\title{
Disinhibition of masking in auditory sensory memory
}

\author{
ROBERT G. CROWDER \\ Yale University, New Haven, Connecticut 06520
}

\begin{abstract}
A series of experiments was performed on the difference between single- and double-masking agents in auditory memory. Single or double suffixes were presented following immediate memory lists, with parametric variation in the delay of the suffixes relative to the end of the list. The main interest was in the shape of the masking function produced by the timing of either the single suffix or the second of two suffixes. Disinhibition was shown to occur, although it was weak in absolute magnitude.
\end{abstract}

The purpose of this report is to provide further information on the occurrence of disinhibition in auditory memory. Disinhibition is a term that describes a particular experimental result that occurs when a second interfering or masking event leads to better performance on some target information than would have been obtained with only a single mask. Crowder (1978) reported disinhibition in immediate memory after finding that a series of three suffixes (extra words) following auditory memory-span lists led to better performance on the last list item than did only a single suffix. This finding was interpreted within the framework of a model for auditory memory that assumes a grid-like representation following rules for lateral inhibition. In the sections that follow, other references to disinhibition in psychology will be reviewed, and then the Crowder (1978) model will be described.

\section{Disinhibition in Cognitive Psychology}

The theoretical and empirical status of disinhibition has been worked out very completely for the retinal cells of the horseshoe crab (Ratliff, 1965). These retinal cells form a two-dimensional grid in which it is possible to deliver light stimuli to, and record electrical activity from, individual cells. Disinhibition is a property of a certain form of lateral inhibition. Therefore, the first step in explaining disinhibition is to describe how lateral inhibition works.

For lateral inhibition, the important pattern of results is that the firing of a unit to stimulation is reduced when a neighboring unit is also being stimulated at the same time. This lateral inhibition is explained by the assumption that units send not only excitatory messages to the next stage of organization, but also inhibitory messages

This research was supported by Grants BNS77-07062 and BNS 8005838 to R. Crowder and by NICHD Grant HD01994 and NSF Grant BRS RR05596 to Haskins Laboratories. Experiments 1 and 2 were conducted by Jeffrey Friedman when he was a senior at Yale University. Georgia Nigro collected the data for Experiment 3. I acknowledge with pleasure the assistance of Virginia Walters in all phases of this research program.

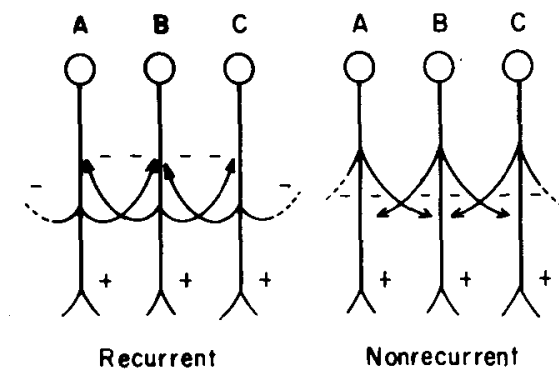

Figure 1. Nonrecurrent and recurrent lateral inhibition networks. Unit $A$ is considered the target and Units $B$ and $C$ the masks.

to neighboring units at the same stage. The degree of lateral inhibition is related to how far the two units are from each other. At very short distances, the two units' activities seem to combine rather than to inhibit each other. At great distances, two units behave independently, that is, one unit responds the same to its stimulation whether or not there is another active unit at a distance. The greatest lateral inhibition is found at an intermediate spacing on the retinal mosaic. It is not important what these distances are in real units; the important point is the inverted U-shaped masking function based on the distance of the target and masking cells.

Figure 1 shows two forms of lateral inhibition for three hypothetical units, A, B, and C. These units are simultaneously emitting excitatory impulses $(+)$ to the next level and also inhibitory impulses $(-)$ to each other. In both types of lateral inhibition, nonrecurrent and recurrent, the firing of $\mathrm{A}$ will be reduced by the simultaneous activity of B. However, there is an important difference between the two inhibitory circuits, a difference that is fundamental to the concept of disinhibition. In nonrecurrent lateral inhibition, the damage to one unit caused by the other is not related to how much the first unit has itself been inhibited. That is, the amount that $A$ is inhibited by $B$ depends only on how active $B$ is before being inhibited by $A$. In recurrent lateral inhibition, the amount of damage that $B$ 
can cause $\mathrm{A}$ already reflects the damage that $\mathrm{A}$ has caused B. In other words, in the recurrent model, the inhibitory effect of one unit impinges on a neighbor above the point at which the neighbor branches out and sends inhibition back to the original unit.

Disinhibition is a property of recurrent, but not of nonrecurrent, lateral inhibition. To see this, consider a third unit, $\mathrm{C}$, in Figure 1, connected to $\mathrm{A}$ and $\mathrm{B}$ according to either arrangement. Assuming our interest is in the firing of Unit A, we can add activity in B, noting a reduction in the activity of $A$. This is the case with either arrangement from Figure 1 and it establishes that $A$ and $B$ are related by some form of lateral inhibition. The next question is what will happen as a consequence of making the third unit, $\mathrm{C}$, active. In nonrecurrent lateral inhibition, the activity in $\mathrm{C}$ will certainly reduce the output of $B$, but it will not influence the amount of inhibition coming from B to $A$. This is because the inhibition fed by $B$ to $A$ has already been sent out before the unit $C$ contacts $B$. In recurrent lateral inhibition, however, the activity of $C$ will inhibit $B$ before $B$ has sent out its inhibitory influences. This means that $\mathrm{C}$ will reduce the ability of $\mathrm{B}$ to inhibit $\mathrm{A}$. Thus, with recurrent lateral inhibition, a mask applied to a mask ( $C$ applied to $B$ ) should increase activity of the target (A). This is the defining outcome for disinhibition.

The limited, scattered literature based on these ideas in psychology encompasses three broad approaches to applications of the model: electrophysiological, theoretical, and behavioral. In the auditory domain, electrophysiological work by Galambos and Davis (1944) established analogues of the "receptive fields" that were later demonstrated by Hubel and Wiesel (1962) in cats' visual systems. The center-surround organization of these networks includes the same logic outlined above and verified for the horsehoe crab retina.

Theoretical explorations of the lateral-inhibition and disinhibition ideas have included abstract investigations of the mathematical properties of systems following the Ratliff (1965) equations (Berman \& Stewart, 1978) and also some psychological theorizing. Milner (1957) found it necessary to include lateral inhibitory assumptions in his realization of Hebb's cell assembly theory, for example. More recently, Walley and Weiden (1973) have offered a theory of selective attention deriving from concepts of lateral inhibition.

In human perception, there have been at least two areas in which disinhibition as the name of an experimental result has been observed. In visual masking, reports by Robinson (1966) and Dember and Purcell (1967) established disinhibition in tachistoscopic research. In one kind of experiment, a faint disk can be inhibited by a surrounding ring but disinhibited by a second ring that surrounds the first ring. There continues to be a lively interest in this phenomenon (e.g., Bryon \& Banks, 1980; Turvey, 1973). However, an isolated report by Deutsch and Feroe (1975) is most relevant to the issues at hand because it shows disinhibition within the domain of auditory short-term memory. The Deutsch and Feroe experiment will be considered in some detail in order to set the context for the present research.

The Deutsch and Feroe (1975) study. Deutsch and Feroe asked subjects for same-different judgments on pairs of tones (a standard and a comparison tone) that were either identical or were .5 whole-tone steps apart. (A whole-tone step is equivalent to two keys on the piano separated by exactly one other key, without regard for black or white. In terms of hertz, the ratio of notes a whole-tone step apart is $1.125: 1.000$.) To make the task nontrivial, they interpolated six interference tones between the standard and the comparison. The interpolated tones never came within 1.5 whole-tone steps of the standard in their baseline or control condition.

In one experiment, the second of the six interference tones was allowed to come close to the standard and comparison tones, however. This critical interference tone was either, in different conditions, the same as the first (standard) tone, or $1 / 6,2 / 6,3 / 6,4 / 6,5 / 6$, or $6 / 6$ of a whole-tone step away from it. Thus, the second interference tone was deliberately made similar to the standard and comparison tones.

The results of this comparison can be described in terms of errors on "same" trials. When the critical second interference tone was identical to the standard (and also identical to the comparison, since only "same" trials are under consideration), performance was better than in the control condition, in which all six of the interference tones were at least 1.5 steps away. In the other conditions, there was an inverted U-shaped masking function: Performance was worst when a 4/6 wholetone step separated the second interference tone from the standard. When the separation was a whole tone (6/6 step), performance was not different from the control condition, nor was it different when only a 1/6-step separation was used. These results are shown in the lower function of Figure 2. In other words, the most interference occurred at an intermediate separation of the mask and standard target. This outcome fits the typical pattern for lateral inhibition, with most masking at an intermediate spacing of target and mask along some relevant distance dimension. Here, however, the dimension is tonal distance rather than spatial distance on the retinal mosaic.

In the next experiment, Deutsch and Feroe made both the second and the fourth of the six interfering tones similar in pitch to the standard. For this arrangement, the standard and the second and fourth interfering tones are being considered as a target and two masks. The second interfering tone was fixed at a 4/6-tone separation, the interval that produced the most interference in the previous comparison. The fourth interfering tone in this new experiment was varied in pitch relative to the second interfering tone in the same degrees used before: 


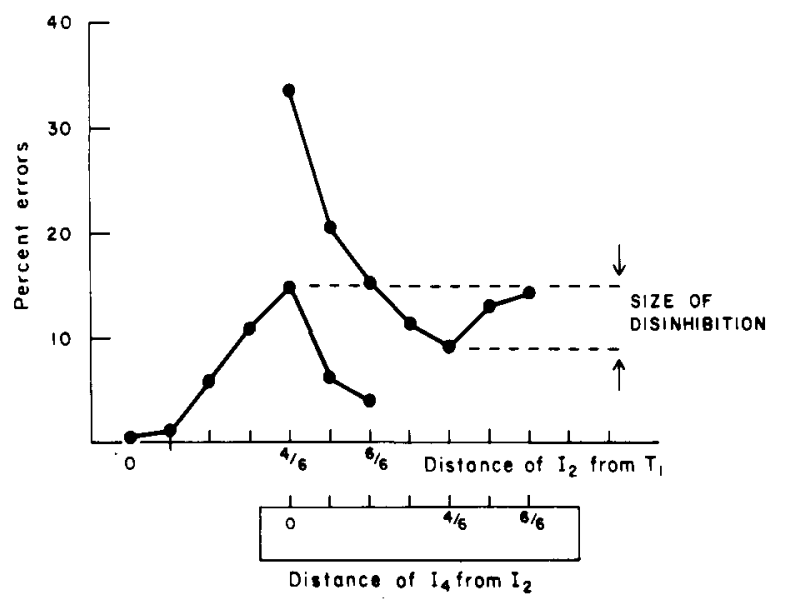

Figure 2. Results of the Deutsch and Feroe (1975) experiment. The performance measure is errors on "same" trials, given as a function of the tonal separation between tones. In the lower function, the separation is between the standard tone (T1) and a single interfering tone (12); in the upper function, the separation is between the first and second of two interfering tones ( 12 and $I 4$, respectively).

$0,1 / 6,2 / 6,3 / 6,4 / 6,5 / 6$, and $6 / 6$ whole-tone steps apart.

The logic behind the second experiment of Deutsch and Feroe was that the fourth tone in the interference series should mask the second tone in the interference series. This masking should be strongest at the same separation (4/6 tone) that produced the strongest masking between the second interfering tone and the target. However, one cannot observe masking going on among interference tones directly. The only performance measure is the same-different response on the comparison tone. Provided the system operates according to recurrent lateral inhibition, however, there is a prediction to be made relative to performance on the same-different task: The effect of double masking (both the second and fourth of the interfering tones) should occur in the form of disinhibition, with the fourth interfering tone producing better performance on the standard tone than would have occurred with only the second interfering tone operating. This would be because the fourth interference tone would inhibit activity of the second interference tone and the second interference tone would thereby be less able to inhibit the target.

Figure 2 (upper function) presents the DeutschFeroe results for the double-masking conditions. Several aspects of the results are noteworthy. First, in general, having both the second and fourth interfering tones close in pitch to the standard produced more errors than having only the second one close in pitch. This seems, on the face of it, to represent the opposite to disinhibitiontwo masks' leading to worse performance than one. One might have insisted that disinhibition would be shown only to the extent that a double-masking condition led to better performance than a single-masking condition.
However, that conclusion would be premature. The real question is whether, when the distance separating the second interfering tone from the target is fixed at $4 / 6$ of a whole-tone step, performance gets better or worse when the fourth interfering tone is set up to interfere with the second. Thus, the relevant point from the single-mask curve is the one at 4/6-step separation, and that point is to be compared with those on the doublemask curve of Figure 2. Of the latter points, it is the 4/6-step separation between the second and fourth interfering tones that is of greatest interest, and there is an unambiguous "absolute" disinhibition effect. Furthermore, the functional relationship between mask delay and performance is precisely opposite for the doubleand single-mask conditions. Whereas inhibition in the single-mask conditions was an inverted U-shaped function of mask delay, there is a U-shaped function when one considers the timing of the second of two masks.

Deutsch and Feroe's experiment thus demonstrates disinhibition in auditory short-term memory. Presently, the result will be rationalized within a theoretical context that draws on ideas of lateral inhibition from sensory psychology, but this is a form of cognition that is obviously "higher" than the retina of the horseshoe crab. The second lesson of this experiment is that the important signature of disinhibition, empirically, is as much (or more) the functional relation between performance and delay in single- and double-mask conditions as it is the simple observation of better performance with double than with single masks. The experiments to be reported below are similar in logic and design to the Deutsch and Feroe experiments.

\section{A Model for Disinhibition in Auditory Memory}

Figure 3 presents a schematic model based on assump. tions made by Crowder (1978; see also Crowder, 1981, 1982). The grid symbolizes a two-dimensional memory representation for auditory events. Entries are classified

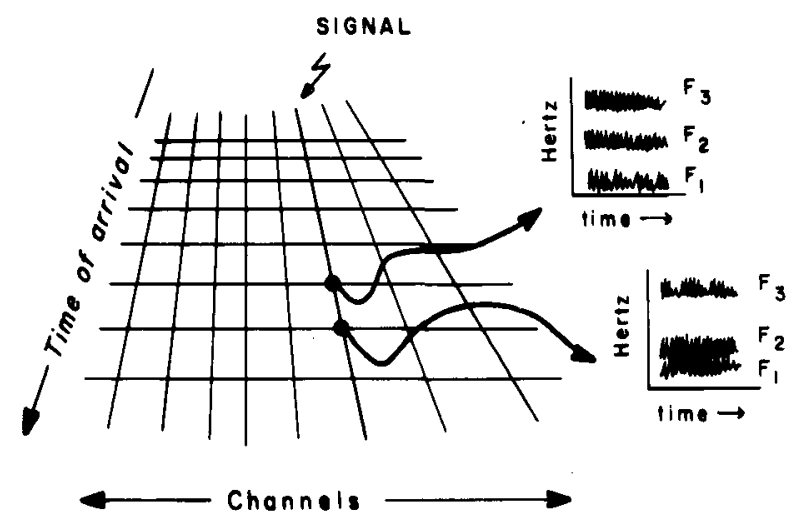

Figure 3. A representation for auditory memories in twodimensional neural space. Entries are classified by channel of entry and time of arrival. The entries themselves are equivalent to rough spectrograms. 
by time of arrival and by "channel." At this point, the definition of "channel" remains unclear. Words spoken by two different speakers would come over different channels, the more so if the two speakers were of different sexes. Words from the same speaker, but located differently in auditory space, would be entered on different channels as well. The channel separation of a speech sound and a nonspeech sound (tone) would be extremely large compared with differences among speech channels. Changes in pitch or stress from a single speech source might or might not produce functional channel separation. In any case, it is quite easy to accept that a single source remains ordinarily on one channel and that the classic operations of selective attention for channel separation (voice quality, location, and so on) result in multichannel stimulation. That much granted, there is no need at the present level of development of the theory to be obsessed with the exact defining features of channel differences.

The model assumes that distinctions in time of arrival and channel are registered in a neurally spatial form, and that there is some sense in which information arriving at different times "goes to different places," as does information arriving over different channels. This twodimensional memory array obviously sets the stage for applying the ideas of lateral inhibition, which depart from the two-dimensional array formed by cells in the retina.

So far, the grid model specifies only that an auditory event will produce activation of some kind at the intersection formed by its arrival time and source channel. For the representation to be useful in a functional sense, it should also provide information about what occurred at a particualr time on a particular channel. As Figure 3 indicates, this problem is addressed by the assumption that grid entries consist of crude spectrograms of the auditory event in question. The idea of a sensory store holding spectral information for auditory events is also a feature of Klatt's (1980) speech perception model.

Thus, Figure 3 models the state of the auditory memory system following presentation of two steadystate vowel sounds distinguished by their second-formant frequencies, both occurring on the channel marked "signal" and occurring one after the other. It is assumed that entries like those shown in Figure 3 operate according to the rule of recurrent lateral inhibition. Specifically, this means there should be an inverted U-shaped masking function relating the masking effect of one entry upon another as a function of the euclidian distance between them, distance either in source channel or in time of arrival. Furthermore, if the form of lateral inhibition is indeed recurrent, a second masking stimulus should degrade the first masking stimulus in a way that produces disinhibited performance on the target item.

Application of the grid model to the Deutsch and Feroe (1975) experiment. In Figure 4, the experiment of Deutsch and Feroe is schematized in terms of the grid model. T1 and T2 stand for the standard and comparison tones, respectively; I 2 and I4 stand for the second

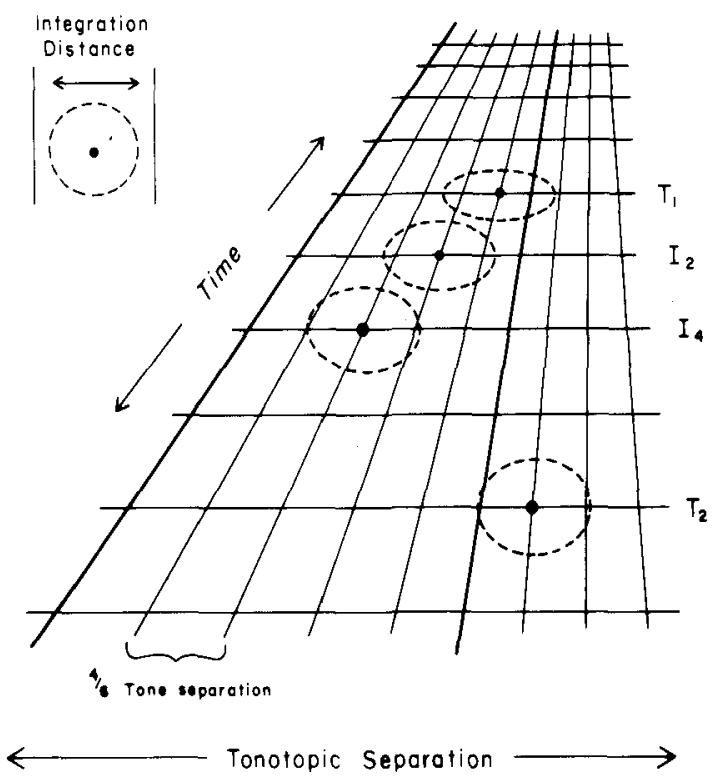

Figure 4. The Deutsch and Feroe (1975) experiment described in terms of the model in Figure 3. T1 and T2 refer to the standard and comparison pitches, respectively. $11,12, \ldots 16$ represent the six interfering tones interpolated between $\mathrm{T} 1$ and $\mathrm{T} 2$.

and fourth in the series of the six interfering tones (the other interfering tones were distant enough to be out of the picture). The only significant change is a simplification of the model to the effect that the dimension of pitch is substituted for channel. It seems reasonable that, in a context in which only tones differing in pitch can occur, the tonotopic organization would stand for channel differences. One can imagine the tonotopic organization of Figure 4 as an expanded "blowup" of just one segment of the larger channel dimension represented in Figure 3. The model of Figure 4 is simpler, furthermore, because the information contained at one of the grid intersections need only be a unidimensional activation. In this sense, the analogy to the visual system is much closer: Information in the network is only that a particular location was active.

From the Deutsch and Feroe result of Figure 2, it can be seen that pitch separations of $1 / 6$ or $2 / 6$ wholetone steps lie within the integration zone of the representation of the standard. Separations of $5 / 6$ or $6 / 6$, on the other hand, lie beyond the reach of the lateral inhibitory connections. Shown in Figure 4 are 4/6step separations of the standard from the second interfering tone and of the latter from the fourth interfering tone.

Application to the suffix experiment. The major point in Crowder (1978) was application of the Figure 3 model to the stimulus suffix experiment. Briefly, the reasoning is that each of the memory list items gets entered, as it is heard, at the appropriate intersection of arrival time and input channel. By the time the end of the list comes, a process called "damped oscillation" (Cornsweet, 1970) will have reduced the potency of the entries for the early list positions, and therefore it is legitimate to restrict attention to the final end of the 
list. Baddeley and Hull (1979) and Engle (1980) have recently provided solid evidence that the last serial position is the only place to look, in modality and suffix experiments, for evidence relevant to auditory sensory memory.

In the situations of interest here, the information all comes in over a single channel. (Although one could argue that different spoken items carry spectral information that varies like the tones of the Deutsch and Feroe, 1975, experiment, the important channel determiner may be the speaker's fundamental pitch and not the changing formant structures.) In the control condition of the suffix experiment, there is either no item following the last memory stimulus, or there is a recall cue on another channel (a buzzer or tone) that is so far removed in the grid that it might as well not have occurred for purposes of the auditory store. (Of course, that does not mean it is ignored by the subject. Two stimuli can quite well be out of reach on the auditory memory representation but wind up in a common working memory store.) Of the last few items, then, the final one should have an especially strong representation on the grid because it is receiving inhibition from only one, rather than two, directions. When a suffix is added on the same channel as the memory list, it is the suffix that receives the benefit of this edge-sharpening process: Now the final memory item is, like the other memory items, getting inhibition from both of the two items neighboring it. Disinhibition occurs when a second suffix is added after the first suffix, for the reasons explained above.

The focus from which the present research derives is the set of predictions for inhibition as a function of grid separation between the last memory item and one or two suffixes. Grid separation will be operationalized here as time separation rather than as channel separation. There are quite a few published experiments on the timing of the suffix. Crowder (1978, Figure 5, p. 515) presented a composite graph from several experiments varying the time delay between the last memory item and a single suffix from 0 to $2 \mathrm{sec}$. The measure of performance was how damaging the suffix was to the last memory item. The form of the overall function was an inverted $U$, with maximum interference occurring somewhere between .5 and $1.0 \mathrm{sec}$. We may conjecture that this is analogous to the lower function of Figure 2, the inverted $U$ obtained by Deutsch and Feroe (1975) for a single mask as a function of its separation from the standard. The purpose of the first experiment in this series was to demonstrate this U-shaped function within a single experiment and to estimate the spacing at which a single suffix has its maximum effect. This estimate can then be used to fix the first of two suffixes and test for disinhibition as a function of the spacing between the first and second suffixes.

\section{EXPERIMENT 1}

In this experiment, there were nine conditions, with parametric variation in the time separation of the last memory item from a single suffix. It was expected from previous work (see Crowder, 1978, Figure 5) that there would be an inverted U-shaped function relating the size of the suffix effect to suffix delay. The purpose was to make a numerical estimate of the inflection point of this function at which masking is greatest.

\section{Method}

Subjects. The subjects were 20 paid volunteers of college age. Most were Yale undergraduates and 12 were males.

Design. All subjects served in nine conditions, which varied according to the time delay between the last item in the memory list (nine digits) and the occurrence of the suffix "go." There were 90 trials, 10 each for the nine delay conditions. These were randomized within blocks of nine trials so that no condition repeated itself until all nine had occurred. Two versions of the experiment were prepared: In the second, the memory items were exchanged on a random basis, so that wherever the digit 9 occurred in the first version, the digit 7 might occur in the second version, for example, and so on. However, the order of suffix delay conditions was the same for the two versions. This meant that performance in a given condition was based on a total of 20 different nine-digit stimuli.

Materials. The nine digits, the word "go," and the word "ready" were recorded by a male speaker. They were then digitized by the Haskins Laboratories pulse code modulation system and stored in computer files. Other routines were then available for sequencing these utterances in specified timing relations and synthesizing them on audiotape. These procedures assured that a given utterance sounded identical regardless of the list or experimental condition in which it occurred. Experiments of this sort are basically impossible without these precautions, for the prosodic output of a real-time speaker is quite likely to be affected by the same variables as those tested as experimental manipulations in suffix experiments.

Each of the digits and the word "go" were placed in a $500-\mathrm{msec}$ frame in such a way as to be roughly "P-centered" (Morton, Marcus, \& Frankish, 1976). No effort was made, however, to correct the natural tendency for some digits to be spoken faster than others, so there was some variation among them in the amount of silence. A 100-msec gap was placed between all adjacent items on the test tape. Thus, it sounded as if the list were being spoken rhythmically at a rate of $600 \mathrm{msec} / \mathrm{item}$.

A trial began with the word "ready," followed by a gap of $500 \mathrm{msec}$ and the nine digits, set at a stimulus onset asynchrony of $600 \mathrm{msec}$. The stimulus onset asynchrony of the ninth mem. ory item relative to the suffix was varied in 100 -msec steps from 100 to $900 \mathrm{msec}$. To accomplish this, the memory items were recorded on one channel and the suffix item was recorded on the second channel of a stereo tape recorder. Fifteen seconds were allowed after the suffix, for written recall, before the next ready signal occurred.

Procedure. The stimuli were presented to subjects who were tested in small groups (one to five individuals) over loudspeakers placed at different sides of the room. How loud the materials seemed depended on where the subject sat, as did, to a slight extent, the relative loudness of the memory items and suffixes (see Crowder, 1978, for data on the importance of these factors). In any case, the memory items and the suffix were on "different channels" with respect to the model of Figure 3.

The instructions called for written, ordered recall. The subjects were told that the suffix "go" was a signal telling them when to write down the nine digits. Opposite each trial number was a set of nine blanks that were to be filled in from left to right, with no backtracking. If the subject failed to remember what went in a position, he was to draw a dash in that space. There was a 2-min break halfway through the session. 

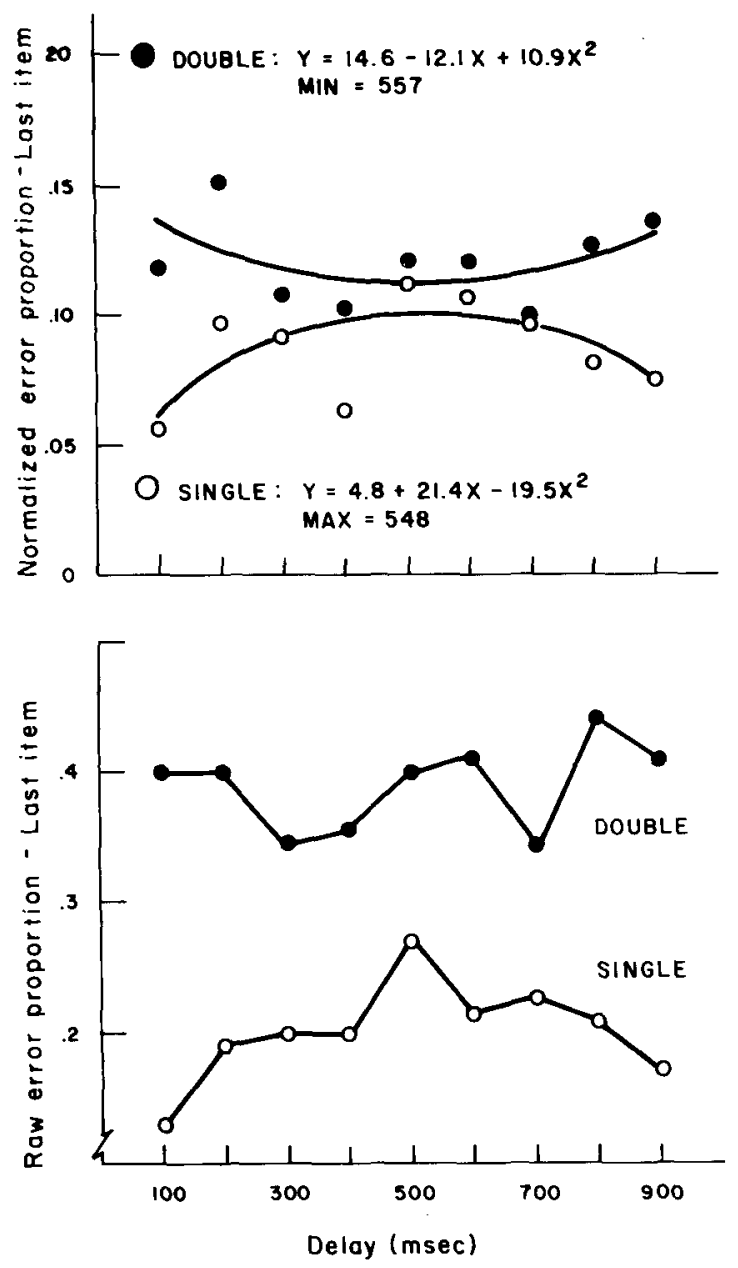

Figure 5. Performance on the ninth serial position as a function of delay for a single suffix (lower curve) or delay for the second of two suffixes (upper curve). In the latter case, the first suffix was fixed at a delay of $500 \mathrm{msec}$.

\section{Results}

Figure 5 shows the results, in the lower curve in each panel, marked "single." In the upper panel are the normalized proportion of errors on the final serial position. For each subject, the errors on the last position in each condition were divided by the total number of errors made on all positions in that condition. In the lower panel, the raw number of final-position errors is given. The normalized errors are the more analytical scores, because they discount the operation of variables that influence performance all across the list rather than just at the end. The layout of Figure 4 (and of the others in this series) is different from that used by Deutsch and Feroe (1975) (see Figure 2) only in that the doublemask curve has been shifted to the left in order to lie over the single-mask curve.

Clearly, the single-suffix data show the predicted inverted-U form, with the largest effect at an intermediate delay. An overall one-way analysis of variance was conducted prior to testing for trend. The result is given in the first row of Table 1 in the column labeled "Overall F." In fact, the reliability of this analysis was borderline $[F(8,152)=1.92$, $\mathrm{MSe}=3225.4, \mathrm{p}<.10]$; however, a glance at Table 1 shows that Experiment 3 of the present series yielded a reliable $F$ for this particular comparison. Furthermore, the obtained function was the one predicted. Trend analyses of the first four degrees are also shown in Table 1 , in which it can be seen that the expected quadratic component was highly significant. The best-fitting quadratic function, obtained by a least squares method, is shown in the upper panel of Figure 5 for these data. The fitted function reaches a maximum of $548 \mathrm{msec}$.

\section{Discussion}

The results of Experiment 1 give the information needed for continuing with double-suffix conditions: The quadratic function relating single-suffix delay to performance was amply confirmed with a relatively small number of observations. Although there was no need for a no-suffix control in this experiment, the obtained magnitude of the suffix effects in Figure 5 was rather smaller than that found in studies using comparable techniques. This is almost surely a result of having placed the suffix and memory items on different loudspeakers and having given them different spatial sources. This channel separation is expected, from the model of Figure 3, to reduce the suffix effect overall. It was built into the design of Experiment 1 in order to minimize direct, integration masking of the memory item by the suffix (see Crowder, 1978). In any case, the magnitude of the suffix effect was not at stake here, only its dependence on the suffix delay.

\section{EXPERIMENT 2}

The second experiment used two suffixes, the first fixed at a delay of $500 \mathrm{msec}$ in all conditions. The purpose was to see whether the relation between the second.

Table 1

Statistical Summaries of Experiments 1-4: Normalized Error Proportions

\begin{tabular}{ccccccc} 
Experiment & $\begin{array}{c}\text { Number of } \\
\text { Suffixes }\end{array}$ & Overall F (One-Way ANOVA) & Linear & Quadratic & Trend Components \\
\hline 1 & 1 & $\mathrm{~F}(8,152)=1.92, \mathrm{p}<.10$ & 1.33 & $7.28^{*}$ & .00 & .06 \\
2 & 2 & $\mathrm{~F}(8,232)=2.27, \mathrm{p}<.05$ & .91 & 3.61 & 1.33 & .89 \\
3 & 1 & $\mathrm{~F}(8,280)=3.11, \mathrm{p}<.005$ & $9.70^{*}$ & $6.49^{*}$ & $7.42^{*}$ & .47 \\
3 & 2 & $\mathrm{~F}=.75$, n.s. & .47 & 2.48 & .06 & 1.12 \\
4 & 2 & $\mathrm{~F}(8,472)=2.04, \mathrm{p}<.05$ & 3.36 & $5.04^{*}$ & 1.58 \\
\hline
\end{tabular}

${ }^{*} p<.05$. 
suffix delay and performance would be a mirror reflection of the single-suffix performance, as would be expected from the disinhibition assumption. The second suffix was presented at the same nine stimulus onset asynchronies $(100,200, \ldots 900)$ relative to the first suffix as were used in Experiment 1 to separate the single suffix from the last memory item.

\section{Method}

The experiment was similar in all details to Experiment 1 with the following exceptions: The $n$ was increased from 20 to 30 subjects, 19 of whom were males (from the same source as Experiment 1). There were three versions of the same 90 memory trials, produced by isomorphic mapping of individual digits from one version to the next. Ten subjects received each of the three versions. Fianlly, the word "go" was said twice at the end of each list, the first time at a stimulus onset asynchrony of $500 \mathrm{msec}$ and the second time at one of nine stimulus onset asynchronies varying in $100 \mathrm{msec}$ steps between 100 and 900 msec. The memory stimuli and second suffix were recorded on one stereo channel and the first suffix on the other. As in Experiment 1, the two channels were separated by means of loudspeakers placed on different sides of the experimental room. Keeping the memory items and the first suffix on separate channels was intended to reduce integration masking of the last memory item, that is, masking through a process of simple "drowning out." It will be seen in Experiment 4 that these channel differences turned out to be inconsequential in the present type of experiment.

\section{Results}

Figure 5 shows the results of Experiment 2 in the upper functions of both panels. A statistical summary of the outcome is in Table 1 , second row. The overall $F$ was statistically reliable $(\mathrm{p}<.05)$ in this experiment, indicating that the normalized errors on the last position were significantly affected by the placement of the second suffix. The form of the function is weakly curved in the mirror image of the single-suffix function from Experiment 1 . The reliability of the quadratic trend in Experiment 2 was just short of the .05 level of confidence $(F=3.61, p<.10)$. The best-fitting quadratic function is written in Figure 5 for the data of Experiment 2. It is notable that the minimum of this function is very close to the maximum of the function from Experiment 1 (557 vs. 548).

\section{Discussion}

By and large, these data fall into the predicted pattern for recurrent lateral inhibition. Two features of these data are worrisome, however: First, there was no "absolute" disinhibition in the sense that two suffixes led to better target performance than one. Second, the results from the first two studies were not statistically impressive. When the quadratic trend was reliable (Experiment 1), the overall $F$ for conditions was not, and when the latter was reliable, the trend fell just short of statistical significance. For these reasons, further data were collected with very similar experimental procedures.

\section{EXPERIMENT 3}

The third experiment combined Experiments 1 and 2 into a single design. The same stimulus tapes were used as in the earlier studies, but the two loudspeakers were placed side by side, so that all materials came from the same apparent source in both conditions. Thirty-six subjects received the single-suffix tape and another 36 received the double-suffix tape. Within each condition, there were three mappings of individual digits into the basic schedule of memory items.

\section{Results}

Figure 6 shows the results of Experiment 3, plotted the same way as those of Experiments 1 and 2. The statistical outcomes are summarized in Table 1 , third and fourth rows. In the single-suffix condition, there was a highly significant overall $F$ for conditions and significant trends for linear through cubic degrees. The bestfitting quadratic function is shown in the figure; its maximum is $646 \mathrm{msec}$, which is slightly less than $100 \mathrm{msec}$
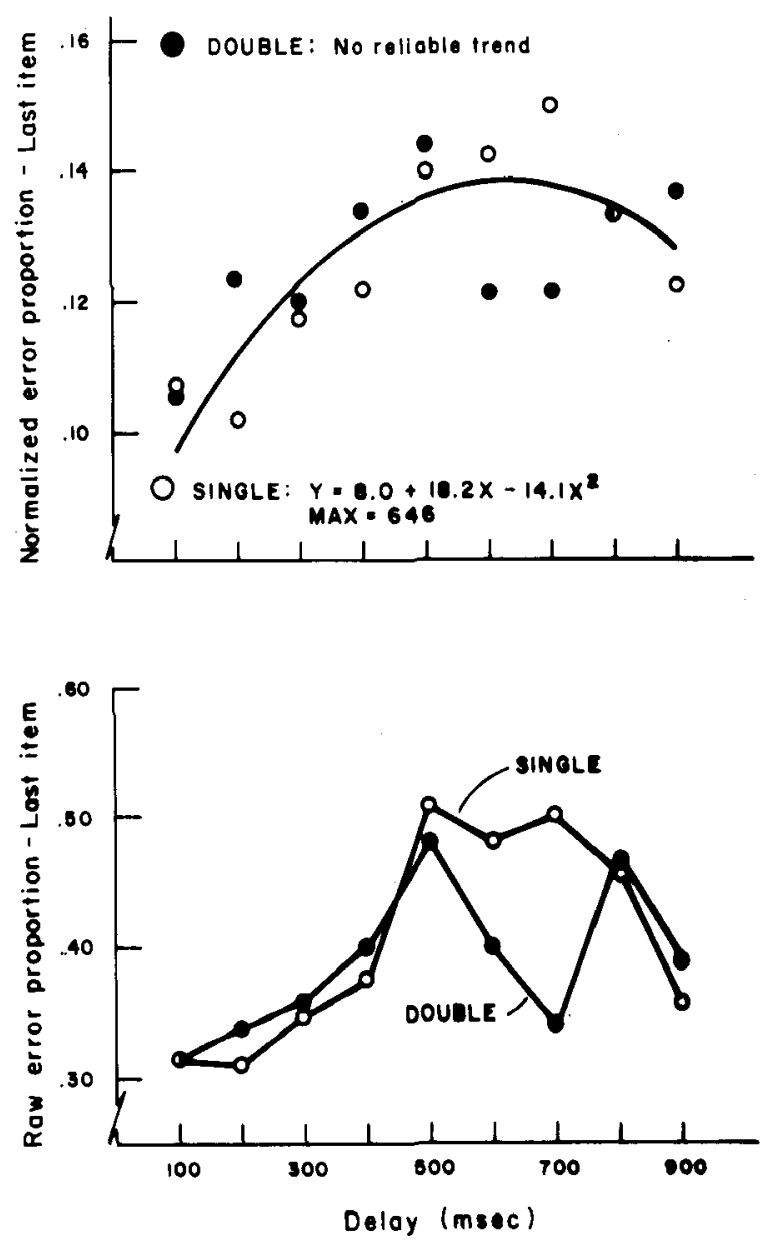

Figure 6. Results of the single- and double-suffix conditions of Experiment 3, plotted the same way as in Figure 5. 
different from the maximum for the function fitted to the single-suffix conditions of Experiment 1.

The results for the double-suffix conditions of Experiment 3 are much less impressive. There was no reliable overall effect of second-suffix delay here, nor was any trend component close to reliability. However, Experiment 3 did show reliable absolute disinhibition: On Positions 6 and 7, performance was significantly better with two suffixes than with one $[t(70)=1.93$, $\mathrm{p}<.05]$. The present experiment is a more appropriate place to look for absolute disinhibition than Experiments 1 and 2 because there was no confounding between suffix number and suffix location and because the subjects were more closely comparable, at least in time of testing. In fact, the results of Experiments 1, 2 , and 3 are really quite comparable if one looks at disinhibition as measured by the difference between normalized last-position errors in the single- and doublesuffix conditions. Such data are shown in Figure 7. The correlation between these two sets of points is +.56 , which shows that both data sets are reliable, and that there is considerable shared variance between them

The restriction of disinhibition to a narrow range in timing of the single- and double-masking events is consistent with what was found by Deutsch and Feroe (1975). In their experiment, absolute disinhibition was obtained only when the maximum of the single-mask condition was compared to the minimum of the doublemask condition (see Figure 2). This also raises a note of caution for experimenters seeking to replicate the effect: Unless these time intervals are delicately calibrated, it is quite likely that one will miss the phenomenon (e.g., Watkins \& Watkins, 1982).

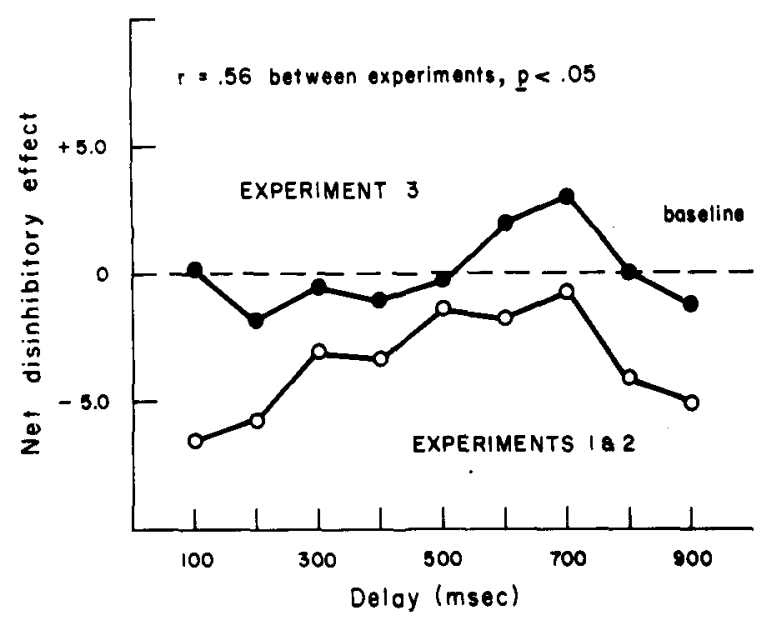

Figure 7. Absolute disinhibition in Experiments 1, 2, and 3. The dependent variable is the algebraic difference, in normalized error proportions on the last serial position, between the singleand double-suffix conditions. The independent variable is the SOA separating the final presented sound (either the single suffix or the second of two suffixes) from the previous one.

\section{EXPERIMENT 4}

The precarious consistency of the statistical evidence from Experiments, 1, 2, and 3 raises still another danger. Perhaps the pattern of Figure 6 is coming entirely from the single-suffix conditions, with the double-suffix conditions serving as little more than baseline controls. The significant overall $\mathrm{F}$ from Experiment 2 and the associated quadratic trend would be considered Type II errors from this viewpoint. The final experiment in this series was an effort to determine whether a U-shaped masking function, with reliable quadratic trend, is "really there" in double-suffix experiments of this type. It was also intended to clear up whether diversity in the spatial sources of the two suffixes makes a difference. In the double-suffix conditions of Experiment 2, the first suffix was on the opposite channel from that which had carried the memory stimuli, and the second suffix returned to the stimulus channel. In Experiment 3, however, all information came over a single channel.

\section{Method}

The method of Experiment 4 was identical to those of the first three experiments except for the following points: Sixty new subjects were used, 30 in each of two groups. In both groups, there were always two suffixes. One group corresponded to the spatial arrangement of Experiment 2 and the other group corresponded to the spatial arrangement of Experiment 3.

\section{Results}

An overall analysis of variance with spatial location of suffixes as one factor (single vs. double source) and second-suffix delay as the other showed no main effect of spatial location or interaction of spatial location with second-suffix delay ( $\mathrm{F}<1.0$ for both). Therefore, the two spatial arrangements have been combined for all subsequent analyses, making this a single-factor, ninecondition experiment. Figure 8 shows the results for normalized last-position errors in the upper panel. The raw errors are shown below, with the single-suffix conditions of Experiment 3 added for comparison. The last row of Table 1 shows that the overall effect of secondsuffix delay was statistically reliable and that the only reliable trend component was the quadratic one. The best-fitting quadratic function has a minimum at $408 \mathrm{msec}$.

\section{Discussion}

The results of Experiment 4 confirmed that the U-shaped masking function for second-suffix delay is not wishful thinking or a false positive. If one wishes to make the comparison shown in the lower panel of Fig. ure 8 between the conditions of the present experiment and the most comparable single-suffix conditions available in this series, there is also ample evidence here for absolute disinhibition. These are the two hallmarks of disinhibition: the mirror reversal of the masking-delay function and the occurrence of absolute disinhibition. 

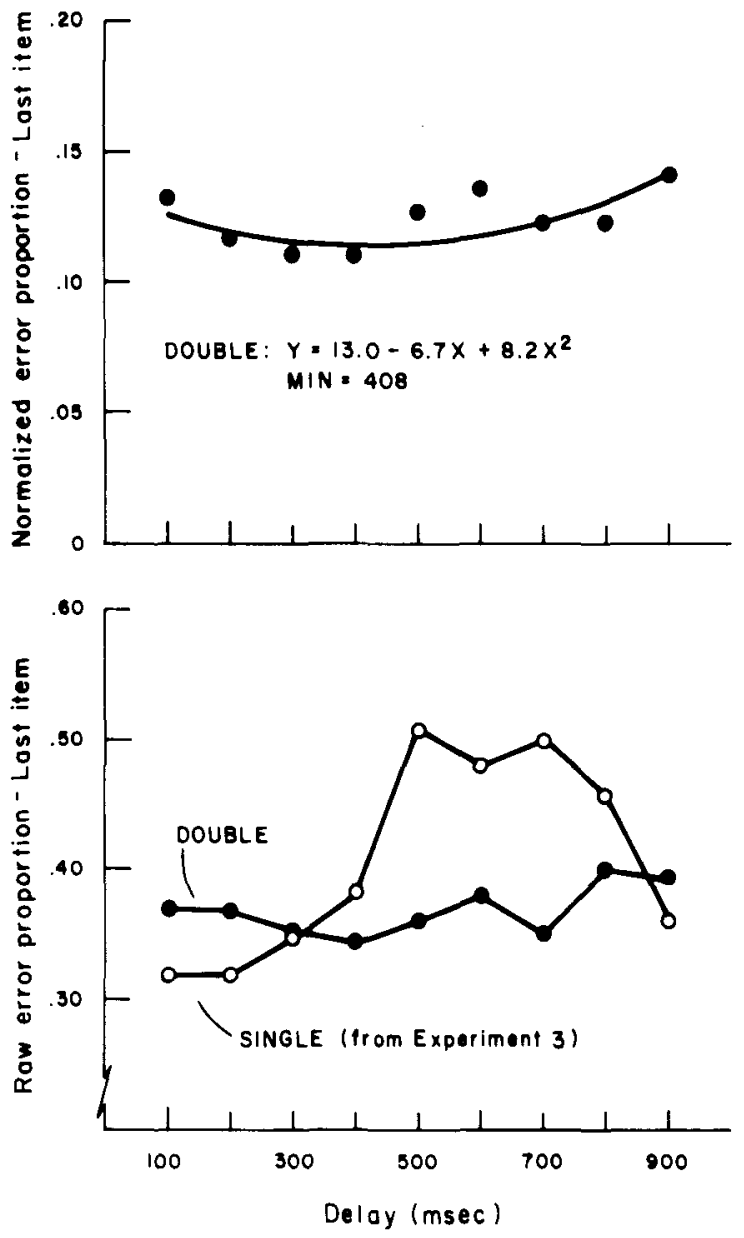

Figure 8. The results of Experiment 4 in the same format as Figures 5 and 6.

There seems to be no reason for retracting Crowder's (1978) hypothesis that suffix experiments can be explained within the grid model and that it is a form of recurrent lateral inhibition that seems to relate entries on that grid.

\section{GENERAL DISCUSSION}

The results of these four experiments differ in statistical impact, and the fitted functions from them show different idiosyncrasies. However, a common theme in them is the predicted quadratic trend. The minima and maxima of the best fitting quadratic functions show a reasonable convergence on something in the neighborhood of $.5 \mathrm{sec}$ as the critical spacing for the strongest lateral inhibition on the grid. There is also some evidence for absolute disinhibition in comparisons of performance in single- and double-suffix conditions.

It could be objected that the results of these experiments depend somehow on using normalized errors on the final position as the main response measure. Watkins and Watkins (1982) have taken strong exception to this practice, for example. One worry might be that the suffix(es) could be affecting items more than one back in the series and, if so, part of the experimental effects might be serving in the normalization background. If so, the argument goes, one's response measure would be tampering improperly with the effect itself. There are many considerations on both sides of this issue. Rather than to weave through these arguments here, an alternative data analysis is offered in Table 2 , which corresponds exactly to Table 1 , except the raw error frequencies on Position 9 were used instead of the normalized proportions. The two analyses show much the same picture. The result of Experiment 2 was not as strong with raw as with normalized errors, and the anomalous result of the double-suffix conditions in Experiment 3 was pushed over the criterion of reliability with the new measure. However, the all important finding of Experiment 4 , which established the $U$-shaped quadratic trend for the double-suffix conditions, was just as convincing in Table 2 as in Table 1. Thus, although normalized errors are stitl the preferred performance index, the conclusions of this research do not change if an uncorrected measure is used.

These experiments show that it is not easy to obtain absolute disinhibition. Only when the timing relations of the two suffixes were exactly right did the doublesuffix condition lead to improved last-item recall. It would not be surprising if other investigations (Watkins \& Watkins, 1982) would have poor luck showing disinhibition if they used only one set of target-mask and intermask delays. Also, it should be noted that the original demonstrations (Crowder, 1978) compared one with three suffixes, whereas the present studies compare one with two. The mathematics of recurrent lateral inhibition networks are complex enough that it is not obvious what the relation should be of double- and triple-

Table 2

Statistical Summaries of Experiments 1-4: Raw Error Frequencies

\begin{tabular}{ccccccc}
\hline & \multirow{2}{*}{$\begin{array}{c}\text { Number of } \\
\text { Experiment }\end{array}$} & Suffixes & Overall F (One-Way ANOVA) & Linear & Quadratic & Cubic \\
\cline { 3 - 7 } & 1 & $\mathrm{~F}(8,152)=1.86, \mathrm{p}<.10$ & .44 & $12.92^{*}$ & .24 & .56 \\
2 & 1 & $\mathrm{~F}(8,232)=1.21$, n.s. & .45 & 1.12 & 1.23 & .00 \\
3 & 1 & $\mathrm{~F}(8,280)=8.83, \mathrm{p}<.0005$ & $22.18^{*}$ & $21.94^{*}$ & $16.96^{*}$ & .23 \\
3 & 2 & $\mathrm{~F}(8,280)=5.06, \mathrm{p}<.0005$ & $8.16^{*}$ & $7.21^{*}$ & .21 & .39 \\
4 & 2 & $\mathrm{~F}(8,472)=2.32, \mathrm{p}<.05$ & $4.46^{*}$ & $4.87^{*}$ & .20 \\
\hline
\end{tabular}

$* p<.05$. 
masking conditions. In the absence of a formal simulation of these outcomes, it remains possible that our understanding of disinhibition is incomplete in this way also.

The magnitude of disinhibition is quite small, however, in these experiments. It would be highly risky to use the amount of disinhibition as an indicator of anything else. Rather, the importance of suffix disinhibition is to settle which type of lateral inhibition, recurrent or nonrecurrent, is the one to use in formal modeling based on the ideas of Figure 3.

Does disinhibition in the auditory system carry implications that go beyond the realm of formal models? It seems likely that a system with the machinery for a sort of temporal edge-sharpening would indeed be important in domains such as speech perception and music. However, these applications should be accomplished with the overall model rather than with the specific assumptions connected with disinhibition.

\section{REFERENCES}

Baddeley, A., \& Hull, A. Prefix and suffix effects: Do they have a common basis? Journal of Verbal Learning and Verbal Behavior, 1979, 18, 139-140.

Berman, S. M., \& Stewart, A. L. The mathematical theory of lateral inpedance of visual activity and an application of Markov processes. Biological Cybernetics, 1978, 30, 99-108.

Byron, D., \& Banks, W. P. Patterned stimuli in disinhibition and backward masking. Bulletin of the Psychomic Society, $1980,15,105-108$.

Corsweet, T. N. Visual perception. New York: Academic Press, 1970.

Crowder, R. G. Mechanisms of auditory backward masking in the stimulus suffix effect. Psychological Review, 1978, 85, 502524.

Crowder, R. G. The role of auditory memory in speech discrimi- nation and perception. In T. Meyers, J. Laver, \& J. Anderson (Eds.), The cognitive representation of speech. Amsterdam: North-Holland, 1981.

Crowder, R. G. Decay of auditory memory in vowel discrimination. Journal of Experimental Psychology: Human Learning and Memory, 1982, 8, 153-162.

Dember, W. N., \& Purcell, D. G. Recovery of masked visual targets by inhibition of the masking stimulus. Science, 1967, 157, 1335-1336.

Deutsch, D., \& Feroe, J. Disinhibition in pitch memory. Perception \& Psychophysics, 1975, 17, 320-324.

EnGLE, R. The suffix effect: How many positions are involved? Memory \& Cognition, 1980, 8, 247-252.

Galambos, R., \& Davis, H. Inhibition of activity in single auditory nerve fibers by acoustic stimulation. Journal of Neurophysiology, 1944, 7, 287-303.

Hubel, D. H., \& Wiesel, T. N. Receptive fields, binocular interaction, and the functional architecture of the cat's visual cortex. Journal of Physiology, 1962, 160, 106-154.

KLATT, D. H. Speech perception: A model of acoustic-phonetic analysis and lexical access. In R. A. Cole (Ed.), Perception and production of fluent speech. Hillsdale, N.J: Erlbaum, 1980.

Milner, P. N. The cell assembly: Mark II. Psychological Review, 1957, 64, 212-252.

Morton, J., Marcus, S., \& Frankish, C. Perceptual centers (P-centers). Psychological Review, 1976, 83, 405-408.

RATLIFF, F. Mach bands: Quantitative studies on neural mechanisms in the retina. San Francisco: Holden-Day, 1965.

Roвınson, D. N. Disinhibition of visually masked stimuli. Science, 1966, 154, 157-158.

TuRvey, M. T. Of peripheral and central processes in vision. Psychological Review, 1973, 80, 1-52.

W ALLEY, R. E., \& WeIDEN, T. D. Lateral inhibition and cognitive masking: A neurophysiological theory of attention. Psychological Review, 1973, 80, 284-302.

Watkins, O. C., \& Watkins, M. J. Lateral inhibition and echoic memory: Some comments on Crowder's (1978) theory. Memory \& Cognition, 1982, 10, 279-286.

(Received for publication July 20, 1981; revision accepted April 3, 1982.) 